\title{
Investigation of Sarcocystis spp. in slaughtered cattle and sheep by peptic digestion and histological examination in Sulaimani Province, Iraq
}

\author{
Shadan Hassan Abdullah (D) \\ Department of Microbiology, College of Veterinary Medicine, Sulaimani University, Sulaimaniyah, Iraq. \\ Corresponding author: Shadan Hassan Abdullah, e-mail: shadan.abdullah@univsul.edu.iq \\ Received: 01-09-2020, Accepted: 11-01-2021, Published online: 22-02-2021
}

doi: www.doi.org/10.14202/vetworld.2021.468-474 How to cite this article: Abdullah SH (2021) Investigation of Sarcocystis spp. in slaughtered cattle and sheep by peptic digestion and histological examination in Sulaimani Province, Iraq, Veterinary World, 14(2): 468-474.

\begin{abstract}
Background and Aim: Sarcocystosis is a zoonotic infection caused by various species of Sarcocystis organisms with a worldwide geographic distribution. This study investigated the presence of Sarcocystis organisms in cattle and sheep slaughtered at an abattoir in Sulaimani Province in North Iraq.

Materials and Methods: A total of 130 muscle samples were collected during May, June, and July of 2020, including 80 samples from sheep and 50 samples from cattle. Samples were examined visually for macrosarcocysts. The peptic digestion method was used to analyze fresh muscle tissue samples for detecting microsarcocysts followed by microscopic examination. Furthermore, muscle samples were fixed and stained with hematoxylin and eosin for histopathological examination.

Results: In the gross examination, macroscopic cysts were not detected in both cattle and sheep; hence, all the prevalence data were obtained through microscopic observation of muscle samples. The peptic digestion method revealed the presence of banana-shaped bradyzoites in $90 \%$ and $92.5 \%$ of slaughtered cattle and sheep muscle samples, respectively. Organ-wise prevalence revealed that $95 \%$ and $92 \%$ of esophageal samples of sheep and cattle contained Sarcocystis spp., respectively. Moreover, $90 \%$ and $88 \%$ of sheep and cattle diaphragms were respectively infected. Histopathological examination of tissue sections revealed two morphologically distinct types of microsarcocysts, including thin-walled and thick-walled, in both sheep and cattle.
\end{abstract}

Conclusion: The suspected Sarcocystis spp. were Sarcocystis tenella and Sarcocystis arieticanis in sheep and Sarcocystis cruzi and Sarcocystis bovifelis or Sarcocystis hominis in cattle. Infective stages of different Sarcocystis spp. are widespread in the study area environment.

Keywords: cattle, muscle samples, prevalence rate, Sarcocystis, sheep.

\section{Introduction}

Sarcocystosis is caused by unicellular coccidian protozoan parasites belonging to Sarcocystis species [1,2]. This genus consists of more than 189 species with global distribution [3]. The majority of the known Sarcocystis spp. appear to be intermediate host-specific [4].

A wide range of vertebrates, including mammals, birds, and fish, are infected by Sarcocystis spp. Merogony and cyst formation (asexual stage) occur in the intermediate host, whereas gametogony and sporogony (sexual stages) occur in the definitive host [5].

Definitive hosts are infected through the ingestion of Sarcocystis in muscle tissues, which include carnivorous predators, scavengers, as well as humans. Intermediate hosts become infected through the ingestion of oocysts/sporocysts present in feed or water

Copyright: Abdullah. Open Access. This article is distributed under the terms of the Creative Commons Attribution 4.0 International License (http://creativecommons.org/licenses/by/4.0/), which permits unrestricted use, distribution, and reproduction in any medium, provided you give appropriate credit to the original author(s) and the source, provide a link to the Creative Commons license, and indicate if changes were made. The Creative Commons Public Domain Dedication waiver (http://creativecommons.org/ publicdomain/zero/1.0/) applies to the data made available in this article, unless otherwise stated. contaminated by fecal materials, which typically consist of herbivores and omnivores [4].

Most of the pathogenic Sarcocystis spp. cause disease only in their intermediate hosts. In general, Sarcocystis spp. transmitted by canids or primates are more pathogenic than those transmitted by felids [5].

Although the majority of Sarcocystis infections are asymptomatic for intermediate hosts, acute cases have also been reported with clinical signs of weakness, anorexia, diarrhea, weight loss, muscle twitching, and, in rare cases, death of the infected animal [6]. These symptoms occur during the development of the second generation of intravascular meronts [7]. The common pathological change associated with sarcocystosis in the intermediate host is the development of tissue cysts containing bradyzoites in the muscle tissues. Occasionally, encephalitis may occur in sheep and horses after infection with Sarcocystis ovicanis and Sarcocystis neurona [8].

Humans can be infected with intestinal sarcocystosis through the consumption of raw or undercooked beef, pork, and meat products containing the bradyzoites of Sarcocystis hominis and Sarcocystis suihominis [9]. The transmission of Sarcocystis cruzi to humans has also been reported [10]. However, 
$S$. cruzi has also been reported in sheep. Nevertheless, the possibility of its transmission from sheep to humans requires careful investigation [11].

Infection generally includes transient nonspecific gastrointestinal manifestations. The severity correlates with the number of ingested sarcocysts [1]. The diagnosis of intermediate hosts is commonly possible during necropsy because the disease exhibits either a subclinical or chronic course [12].

It has been reported that sarcocystosis in sheep (Ovis aries) is caused by Sarcocystis tenella, Scolopendra gigantea, Sarcocystis medusiformis, and Sarcocystis arieticanis [13]. Moreover, Sarcocystis moulei and S. cruzi have been reported in sheep [11]. At the same time, cattle (Bos taurus) are an intermediate host for Sarcocystis spp., including S. cruzi, S. hominis, Sarcocystis hirsuta, and Sarcocystis bovifelis [14]. Other species include Sarcocystis sinensis [15]. Recently, Sarcocystis heydorni, Sarcocystis rommeli, and Sarcocystis bovini have also been identified in bovines $[9,14]$.

Sarcocystis spp. can be microscopic, whereas others are macroscopic sarcocysts [13]; macrosarcocysts in striated muscles are large enough to be distinguished by the naked eye, as they occur as elongated cylindrical bodies and milky white-colored cysts embedded in muscular tissues, with length ranging from $<5 \mathrm{~mm}$ to $>10 \mathrm{~mm}$. Two forms of examined macrosarcocysts were present, including fat and thin forms [16]. In sheep, S. gigantea and S. medusiformis form macroscopic sarcocysts that are transmitted by felids, whereas other Sarcocystis spp. transmitted by canines are microscopic [13]. In cattle, mature $S$. hirsuta cysts are macroscopic, whereas cysts of other species are microscopic [4].

Microscopic sarcocysts are generally identified based on the morphology of the cyst wall; $S$. cruzi and $S$. heydorni have thin-walled cysts, and $S$. bovini, S. bovifelis, S. hirsuta, and S. hominis have thick-walled cysts $[4,9]$. The sarcocysts of $S$. tenella have thick cyst walls, whereas those of S. arieticanis have thin cyst walls [17].

The definitive identification of Sarcocystis species requires an electron microscope or a molecular detection method [18]. Molecular techniques have been used to differentiate Sarcocystis species in cattle and sheep $[4,19]$.

Sarcocystosis, due to its severe economic, medical, and veterinary consequences, is considered as a significant public health issue in several countries [20].

Due to the lack of available data on the prevalence of Sarcocystis spp., this study was conducted to investigate the presence of Sarcocystis organisms in meat-producing animals (cattle and sheep) slaughtered at an abattoir in Sulaimani Province in the northern region of Iraq.

\section{Materials and Methods}

\section{Ethical approval}

The study is not based on in vivo experiments or live animals; the sampling was performed following the slaughtering of animals in the slaughterhouse.

\section{Study period and location}

This study was conducted during May, June, and July of 2020. Muscle samples were collected from the diaphragm and esophagus of slaughtered cattle and sheep of different ages and sexes after inspection of meat at the abattoir in Sulaimani Province. The samples were examined for the presence of Sarcocystis spp.

\section{Sample collection}

A total of 130 muscle samples, including 80 from sheep (40 diaphragm and 40 esophagus samples) and 50 from cattle (25 diaphragm and 25 esophagus samples), were collected. Samples were placed in plastic bags, labeled, and transported in a CoolBox to the laboratory for further investigation.

Fresh tissue samples were subjected to gross examination for the presence of macrocysts, and the microsarcocysts were detected using the peptic digestion method, followed by microscopic examination and histological examination of stained muscle samples [21].

\section{Microscopic examination using peptic digestion method}

Muscle samples were minced and subjected to peptic digestion separately using the method described by Dubey et al. [12]. Approximately $20 \mathrm{~g}$ of each minced sample was processed using $50 \mathrm{~mL}$ of the digestion solution, comprising $1.3 \mathrm{~g}$ pepsin, $2.5 \mathrm{~g}$ $\mathrm{NaCl}$, and $3.5 \mathrm{~mL}$ concentrated $\mathrm{HCl}$ in a total volume of $500 \mathrm{~mL}$ of distilled water.

The suspensions were set at room temperature for $30 \mathrm{~min}$ and then filtered using a strainer with gauze. Filtrates were collected in sterile test tubes and centrifuged at $1500 \mathrm{rpm}$ for $15 \mathrm{~min}$, and the sediment was suspended in $0.5 \mathrm{~mL}$ of distilled water [22].

The suspension was examined for the presence of Sarcocystis bradyzoites under a light microscope at $40 \times$. Free bradyzoites were observed as bananashaped bodies. In addition, smears were prepared using droplets from the same solution spread on glass slides, fixed with absolute methanol, and stained with $10 \%$ Giemsa solution for $5 \mathrm{~min}$. Slides were examined under a microscope at $\times 100$ for detecting Sarcocystis bradyzoites [23].

\section{Histological examination}

A total of 16 samples that were positive in the microscopic examination were subjected to histopathological investigation (eight samples from each organ) for both animal species. Slices of muscle samples were preserved, fixed in $10 \%$ neutral buffered formalin, and embedded in paraffin blocks for histological examination. The tissues were cut into 3-5 $\mathrm{mm}$ thick sections and stained with hematoxylin and eosin (H\&E) [24]. Slides were examined for the presence of sarcocysts at $10 \times$ and further analyzed at $40 \times$ and $100 \times$. For the morphological identification 
of Sarcocystis spp., the cell wall structure was observed [25].

\section{Statistical analysis}

Data analysis was conducted using SPSS Statistic Version 19 (IBM, USA). Chi-square tests were used to determine the association between different organs (inspected esophagus and diaphragms in both cattle and sheep). $\mathrm{p}<0.05$ was considered to be statistically significant.

\section{Results}

The overall prevalence rates of Sarcocystis spp. as examined by light microscopy were $90 \%(45 / 50)$ and $92.5 \%(74 / 80)$ in slaughtered cattle and sheep, respectively. There were no significant differences $(p>0.05)$ between the overall Sarcocystis detection rates in the examined host species (Table-1).

Organ-wise prevalence revealed higher positive rates of Sarcocystis spp. in the esophagus than in the diaphragm. Approximately 95\% (38/40) of esophageal samples contained Sarcocystis, and 90\% (36/40) of diaphragm samples were positive in sheep. In cattle also, $92 \%(23 / 25)$ of esophageal samples were positive, and $88 \%(22 / 25)$ of diaphragm samples were positive for Sarcocystis spp. However, there were no significant differences $(p>0.05)$ between the examined organs in both cattle and sheep. The distribution of Sarcocystis spp. in cattle and sheep muscles is shown in Table-1.

In the gross observation, no macroscopic sarcocysts were found in any of the examined muscle samples representing cattle and sheep. This finding may be due to the variation in risk factors associated with the distribution of Sarcocystis infection.

In the microscopic observation, the bradyzoites appeared crescent shaped and had pointed anterior end and rounded posterior end as analyzed using the peptic digestion method (Figure-1).

Histopathological examination of H\&E-stained tissue sections revealed the presence of elongated, spindle-, round-, and ovoid-shaped microscopic tissue cysts packed with banana-shaped bradyzoites (Figure-2). Microscopic observation also revealed variations in the intensity of Sarcocystis spp. invasion, varying from single to numerous cysts (Figure-3).

Regarding the pathological changes caused by Sarcocystis spp., an inflammatory response around several microcysts was detected in the infected muscle fibers accompanied by infiltration of mononuclear cells and degeneration and necrosis of muscle fibers (Figure-4).

Observation of the microsarcocyst wall under the light microscope revealed two morphologically distinct types, including thin-walled and thick-walled Sarcocystis spp., in both sheep and cattle tissue samples (Figures-5-7). Based on the microsarcocyst morphology, the suspected Sarcocystis spp. in this study were identified as $S$. tenella and $S$. arieticanis in sheep and $S$. cruzi and $S$. bovifelis or $S$. hominis in cattle. This finding indicated that more significant numbers of Sarcocystis spp. were of the thin-walled type in both animals. Sarcocysts were found inside the striated muscle cells including the esophagus, and diaphragm (Figure-8).

\section{Discussion}

Sarcocystis is considered as one of the most prevalent livestock parasites, with potential public health importance due to the consumption of undercooked or raw meat. Sarcocystis spp. have been isolated from different animals throughout the world. However, to the best of our knowledge, the present study is the first to investigate Sarcocystis infection among slaughtered cattle and sheep in Sulaimani Province in North Iraq.

Natural infections of sheep by Sarcocystis spp. have been investigated in various countries, with prevalence rates varying from $9.0 \%$ to $100 \%$, depending on the detection method [13]. Meanwhile, no macroscopic cysts were detected in the visual examination in the present study, and the overall prevalence was obtained through microscopic examination of muscle tissues, which revealed prevalence rates of $90 \%$ and $92.5 \%$ of unspecified cysts of Sarcocystis spp. by the digestion method in cattle and sheep, respectively.

Diversity in sarcocystosis prevalence has been reported in different geographical areas; for instance, a prevalence rate of $100 \%$ was reported by Dehaghi et al. [26] and Whaeeb and Faraj [27] in sheep, and

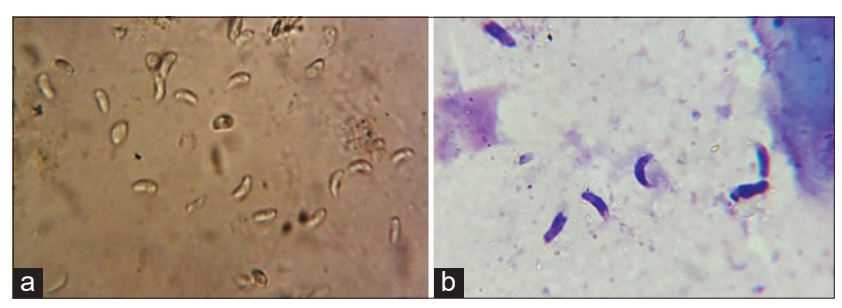

Figure-1: (a) Fresh smear preparation from microcyst, showing crescent-shaped bradyzoites (40x), (b) Giemsa stained from digested muscle samples showing Sarcocystis bradyzoites $(100 \times)$.

Table-1: Prevalence of microsarcocyst of Sarcocysts spp. in slaughtered cattle and sheep at Sulaimani abattoir, north Iraq.

\begin{tabular}{|c|c|c|c|c|c|}
\hline \multirow{3}{*}{$\begin{array}{l}\text { Inspected } \\
\text { organs }\end{array}$} & \multicolumn{4}{|c|}{ Examined animal } & \multirow[t]{3}{*}{ Total infected } \\
\hline & \multicolumn{2}{|c|}{ Cattle } & \multicolumn{2}{|c|}{ Sheep } & \\
\hline & Inspected no. & Infected no. (\%) & Inspected no. & Infected no. (\%) & \\
\hline Esophagus & 25 & $23(92)$ & 40 & $38(95)$ & 65 \\
\hline Diaphragm & 25 & $22(88)$ & 40 & $36(90)$ & 65 \\
\hline Total & 50 & $45(90)$ & 80 & $74(92.5)$ & 130 \\
\hline
\end{tabular}




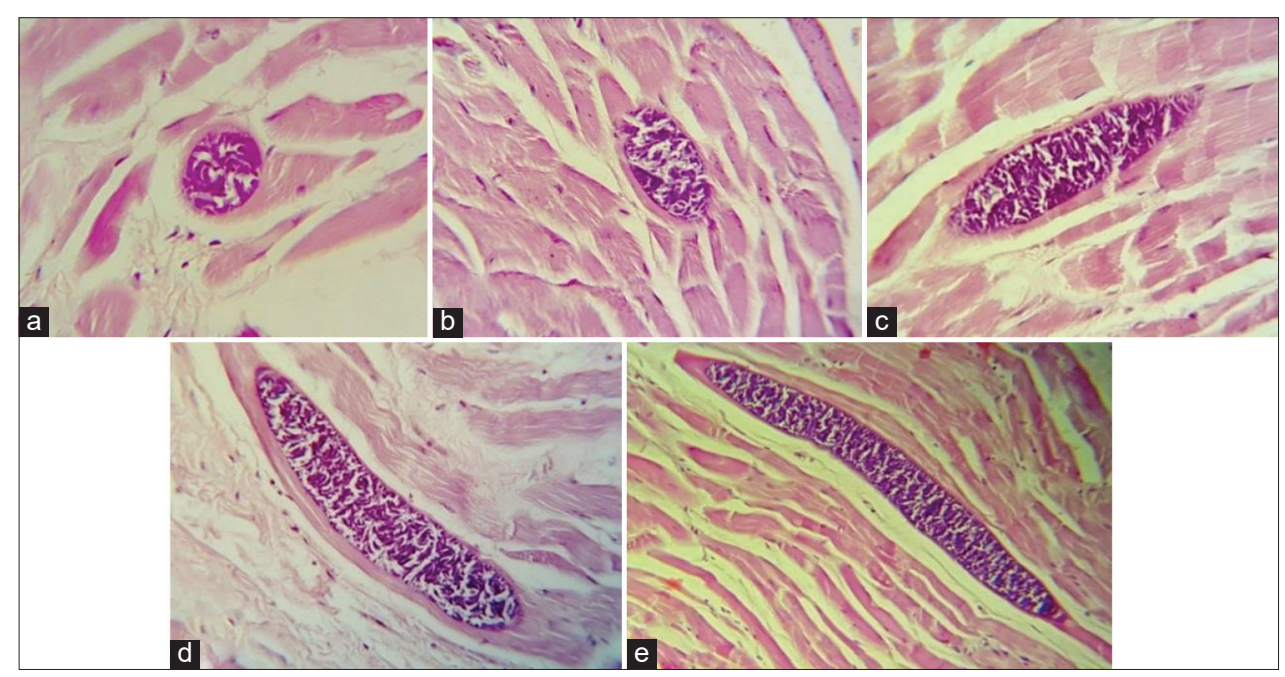

Figure-2: (a-e) Longitudinal section of Sarcocystis spp. revealing different shapes of microsarcocyst with hematoxylin and eosin stain of diaphragm muscles $(10 \times)$.

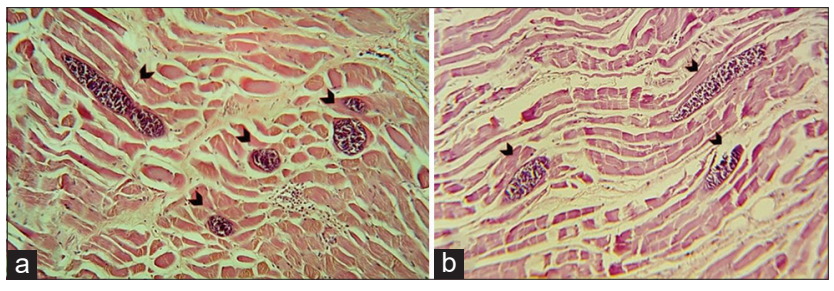

Figure-3: Longitudinal section of unidentified Sarcocystis spp. (a and b) Revealing different microsarcocysts with hematoxylin and eosin stain of diaphragm muscles $(10 \times)$.

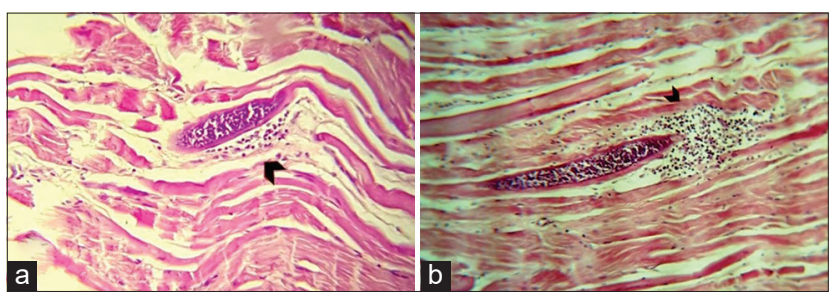

Figure-4: Histological sections from muscle tissue with an unidentified Sarcocystis spp. hematoxylin and eosin stain $(a$ and $b$ ) showing inflammatory reaction $(10 x)$.

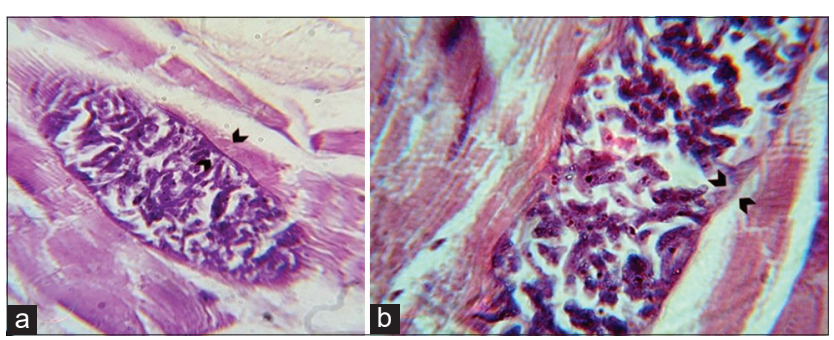

Figure-5: Light microscopic appearance of thin wall microsarcocysts from sheep diaphragm with hematoxylin and eosin stain, (a) $40 \times$ and (b) 100x.

prevalence rates of $95.37 \%$ and $96.9 \%$ were reported by Fukuyo et al. [5] and Abuelwafa [28], respectively.

Similarly, the prevalence in bovine muscle was almost $100 \%$ in most of the world regions [2]. A prevalence of $100 \%$ was reported by Shekarforoush et al. [29]. The prevalence rates of $95.5 \%$, $91.33 \%$, and $88.2 \%$ were reported by Mavi et al. [30], Mounika etal. [31], and Shahraki et al. [32], respectively.

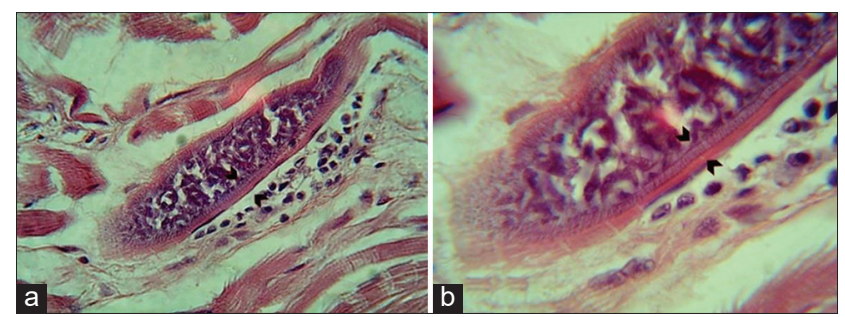

Figure-6: Light microscopic appearance of thick wall microsarcocysts from sheep diaphragm with hematoxylin and eosin stain, (a) $40 \times$ and (b) $100 \times$.

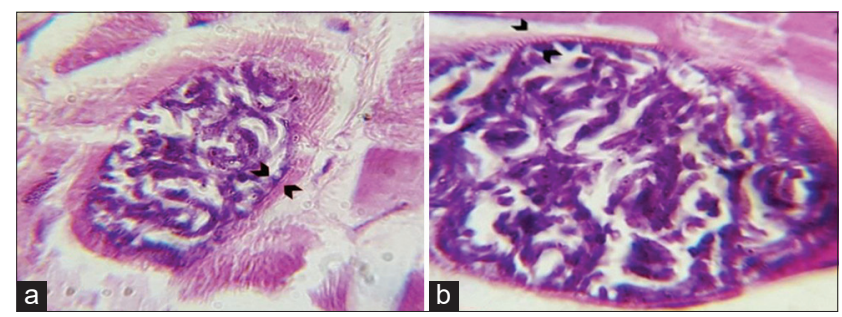

Figure-7: Light microscopic appearance of microsarcocysts from cattle diaphragm with hematoxylin and eosin stain, showing two types of cyst (a) thin wall and (b) thick wall $(100 \times)$.

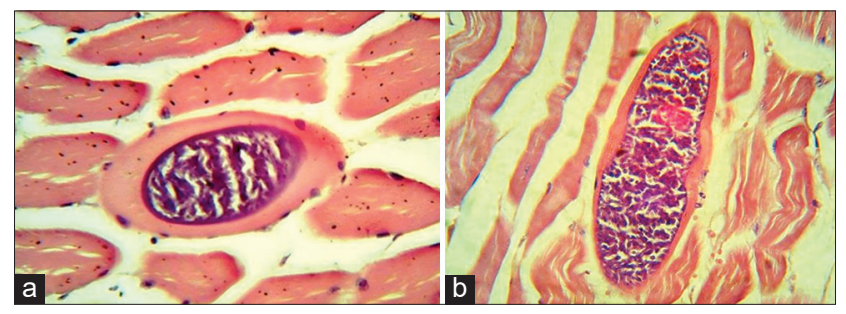

Figure-8: Histological sections of an unidentified Sarcocystis spp. showing the microsarcocysts inside the muscle cells from infected cattle (a) esophagus, (b) diaphragm with hematoxylin and eosin stain (40x).

In the present study, examination of the esophagus and diaphragm muscles revealed higher prevalence rates of $92 \%$ and $95 \%$ in the esophagus than those of $88 \%$ and $90 \%$ in the diaphragm for cattle and sheep, respectively. A similar finding was also reported by Dehaghi et al. [26] and Ahmed et al. [33]. 
Positive rates of $91 \%$ and $58 \%$ in cattle esophagus and diaphragm were also reported by Nourani et al. [34].

In contrast to our findings, high prevalence rates of Sarcocystis spp. of $100 \%$ and $93.2 \%$ in the diaphragm were reported by Fukuyo et al. [5] and Bahari et al. [35], respectively, and also by Dubey and Livingston [20] who reported the prevalence rates of $22.4 \%$ in the diaphragm and $0.23 \%$ in the esophagus of sheep.

Sarcocystis was also detected in cattle heart at prevalence rates of $100 \%$ and $17.9 \%$ from Brazil and Romania by Ferreira et al. [36] and Imre et al. [37], respectively.

Variations in the prevalence rates of Sarcocystis spp. depend on several factors such as management conditions, presence of dogs and cats in the surrounding area, and the number of sporocysts disseminated by them. Survivability of sarcocysts in the environment is another factor affecting the prevalence of Sarcocystis controlled by climatic conditions such as temperature, rainfall, and humidity [38].

The present study results showed that the pepsin digestion technique is a sensitive method for investigating the presence of Sarcocystis in slaughtered animals [32,39], which produced the highest positive rate. This technique is also simpler and faster than other conventional procedures [16]. However, Sarcocystis spp. cannot be appropriately identified using this method.

Similar to the present study result, no macroscopic sarcocysts were observed by Shekarforoush et al. [29], Nourani et al. [34], and Shahraki et al. [32], although low prevalence rates of $17.08 \%$ and $7.5 \%$ were reported by Farhang-Pajuh et al. [16] and Ahmed et al. [33] in sheep and cattle, respectively.

Macrosarcocyst species are considered as symptomatic. However, they can affect the quality of meat and lead to economic losses [19] in the form of subsequent downgrading, partial rejection, or even condemnation of the animal carcasses at slaughterhouses [28].

Primarily, felids comprise the definitive hosts for macrosarcocyst forming species. Due to lesser contact with felids, lesser numbers of oocytes are shed, which thus explain the low prevalence of macroscopic cysts [30].

Dogs play a more significant role than cats in the transmission of sarcocystosis in farm animals, which might be attributed to the close association with dogs that are retained for herd protection. Moreover, dogs can easily access the offal of infected sheep, thus making it easier for them to transmit Sarcocystis [28].

In the present study, histopathological examination of H\&E-stained tissue sections revealed the presence of two morphologically distinct microscopic types of sarcocysts. Consistent with El-Morsey et al. [17], the observed microsarcocysts in sheep muscle tissues were of thin-walled and thick-walled types, which belong to $S$. arieticanis and $S$. tenella, respectively, and those of $S$. tenella had striated thick-walled sarcocysts (Figure-7). Moreover, the bovine species $S$. cruzi has been reported to have thin-walled microsarcocysts detected in sheep [11]. Similarly, both $S$. tenella and $S$. arieticanis were reported through light microscopic examination by $\mathrm{Hu}$ et al. [40].

Sarcocystis spp. in cattle were also of two types. $S$. cruzi and $S$. heydorni have thin-walled cysts, and $S$. bovini, S. bovifelis, and S. hominis have thick-walled cysts [25].

In this regard, based on the morphological appearance, the thin-walled microsarcocysts observed in the present study might be $S$. cruzi and $S$. heydorni. Moreover, the thick-walled type might belong to $S$. hominis, S. bovini, S. sinensis, and S. bovifelis, as these species are very similar morphologically, and their cyst walls are provided with finger-like protrusions [41]. The cysts cannot be distinguished by light microscopy, whereas a few differences can be identified by transmission electron microscopy $[4,6]$.

Based on the histological examination of the microsarcocyst wall, the suspected Sarcocystis spp. were identified as $S$. tenella and S. arieticanis in sheep and $S$. cruzi and $S$. bovifelis or $S$. hominis in cattle, as the availability of other species was inadequate.

Based on the data reported by Nourani et al. [34], the thin-walled sarcocysts in cattle were consistent with $S$. cruzi with higher prevalence, and the thickwalled sarcocysts could represent $S$. hominis with lower prevalence. Both $S$. cruzi and S. tenella were found at higher prevalence rates of $100 \%$ and $80 \%$ by Daryani et al. [20] and Rahdar and Kardooni [42], respectively.

Both intermediate and final hosts can harbor one or more Sarcocystis spp. [28], which is consistent with the present study finding in which both thin- and thick-walled microsarcocysts were observed in the same muscle tissue section.

Sarcocystis spp. cyst invasions in the muscle tissue can produce inflammation around the muscle fibers and between the cysts, showing mononuclear cells infiltration (neutrophils, lymphocytes, eosinophils, and plasma cells) and degeneration and necrosis of muscle fibers [25]. Consistently, inflammatory reactions were also detected around some of the observed microsarcocysts in the present study (Figure-4).

Identification of Sarcocystis spp. is not possible using a microscopic analysis [25]. Therefore, the exact identification and differentiation of Sarcocystis spp. in slaughtered animals necessitate other investigations using accurate detection methodologies, including molecular procedures.

Identification of Sarcocystis spp. through molecular characterization is a fundamental approach to evaluate the pathogenic species involved in cattle and sheep infections that consequently cause economic losses. In addition, identifying the final host responsible for shedding each of Sarcocystis spp. and evaluating the risk of zoonotic infections are essential steps in managing the disease and designing control programs. 


\section{Conclusion}

This study reports the presence of Sarcocystis spp. in both sheep and cattle from Sulaimani Province. The prevalence was primarily related to the presence of microsarcocysts. Therefore, meat should be cooked sufficiently or frozen before use to prevent health hazards to the consumer. The pepsin digestion procedure was found to be a sensitive method for investigating unidentified Sarcocystis spp. Based on the morphology of the microsarcocyst wall, the suspected Sarcocystis spp. recovered in this study were identified as $S$. tenella and $S$. arieticanis in sheep and S. cruzi and S. bovifelis or $S$. hominis in cattle.

\section{Author's Contributions}

SHA designed the study and contributed to the collection of muscle samples, the practical parts, analysis of data, and writing and organization of the paper. SHA has read and approved the final manuscript.

\section{Acknowledgments}

The author would like to thank Dr. Dyary Heiwa from the College of Veterinary Medicine, University of Sulaimani, for his kindness in commenting and improving the manuscript. The author did not receive any funds for this study.

\section{Competing Interests}

The author declares that he has no competing interests.

\section{Publisher's Note}

Veterinary World remains neutral with regard to jurisdictional claims in published institutional affiliation.

\section{References}

1. Fayer, R. (2004) Sarcocystis spp. in human infections. Clin. Microbiol. Rev., 17(4): 894-902.

2. Vangeel, L., Houf, K., Chiers, K., Vercruysse, J., D’Herde, K. and Ducatelle, R. (2007) Molecular-based identification of Sarcocystis hominis in Belgian minced beef. J. Food Prot., 70(6): 1523-1526.

3. Poulsen, C.S. and Stensvold, C.R. (2014) Current status of epidemiology and diagnosis of human sarcocystosis. $J$. Clin. Microbiol., 52(10): 3524-3530.

4. Gjerde, B. (2016a) Molecular characterization of Sarcocystis bovifelis, Sarcocystis bovini n. sp., Sarcocystis hirsuta and Sarcocystis cruzi from cattle (Bos taurus) and Sarcocystis sinensis from water buffaloes (Bubalus bubalis). Parasitol. Res., 115(4): 1473-1492.

5. Fukuyo, M., Battsetseg, G. and Byambaa, B. (2002) Prevalence of Sarcocystis infection in meat-producing animals in Mongolia. Southeast Asian J. Trop. Med. Public Health, 33(3): 490-495

6. Moré, G., Pantchev, A., Skuballa, J., Langenmayer, M., Maksimov, P., Conraths, F., Venturini, M.C. and Schares, G. (2014) Sarcocystis sinensis is the most prevalent thickwalled Sarcocystis species in beef on sale for consumers in Germany. Parasitol. Res., 113(6): 2223-2230.

7. Morsy, K., Saleh, A., Al-Ghamdi, A., Abdel-Ghaffara, F., Al-Rasheid, K., Bashtar, A.R., Al Quraishy, S. and Mehlhorn, H. (2011) Prevalence pattern and biology of Sarcocystis capracanis infection in the Egyptian goats: A light and ultrastructural study. Vet. Parasitol., 181(2-4): 75-82.

8. Formisano, P., Aldridge, B., Alony, Y., Beekhuis, L., Davies, E., Del Pozo, J., Dunn, K., English, K., Morrison, L. and Sargison, N. (2013) Identification of Sarcocystis capracanis in cerebrospinal fluid from sheep with neurological disease. Vet. Parasitol., 193(1-3): 252-255.

9. Dubey, J., van Wilpe, E., Calero-Bernal, R., Verma, S. and Fayer, R. (2015b) Sarcocystis heydorni, n. sp. (Apicomplexa: Sarcocystidae) with cattle (Bos taurus) and human (Homo sapiens) cycle. Parasitol. Res., 114(11): 4143-4147.

10. Agholi, M., Shahabadi, S.N., Motazedian, M.H., and Hatam, G.R. (2016) Prevalence of enteric protozoan oocysts with special reference to Sarcocystis cruzi among fecal samples of diarrheic immunodeficient patients in Iran. Korean J. Parasitol., 54(3): 339-344.

11. Anvari, D., Narouei, E., Hosseini, M., Narouei, M.R., Daryani, A., Shariatzadeh, S.A., Pagheh, A.S., Gholami, S., Sarvi, S. and Sargazi, D. (2020) Sarcocystosis in ruminants of Iran, as neglected food-borne disease: A systematic review and meta-analysis. Acta Parasitol., 65(3): 555-568.

12. Dubey, J., Speer, C. and Fayer, R. (1989) Sarcocystosis of Animals and Man. CRC Press, Boca Raton.

13. Dubey, J., Calero-Bernal, R., Rosenthal, B., Speer, C. and Fayer, R. (2015a). Sarcocystosis of Animals and Humans. CRC Press, Boca Raton.

14. Gjerde, B. (2016b) The resurrection of a species: Sarcocystis bovifelis Heydorn et al., 1975 is distinct from the current Sarcocystis hirsuta in cattle and morphologically indistinguishable from Sarcocystis sinensis in water buffaloes. Parasitol. Res., 115(1): 1-21.

15. Gjerde, B. (2013) Phylogenetic relationships among Sarcocystis species in cervids, cattle and sheep inferred from the mitochondrial cytochrome $\mathrm{c}$ oxidase subunit I gene. Int. J. Parasitol., 43(7): 579-591.

16. Farhang-Pajuh, F., Yakhchali, M. and Mardani, K. (2014) Molecular determination of abundance of infection with Sarcocystis species in slaughtered sheep of Urmia, Iran. Vet. Res. Forum, 5(3): 181-186.

17. El-Morsey, A., Abdo, W., Sultan, K., Elhawary, N.M. and AbouZaid, A.A. (2019) Ultrastructural and molecular identification of the sarcocysts of Sarcocystis tenella and Sarcocystis arieticanis infecting domestic sheep (Ovis aries) from Egypt. Acta Parasitol., 64(3): 501-513.

18. Rosenthal, B.M., Dunams, D.B. and Pritt, B. (2008) Restricted genetic diversity in the ubiquitous cattle parasite, Sarcocystis cruzi. Infect. Genet. Evol., 8(5): 588-592.

19. Kalantari, N., Khaksar, M., Ghaffari, S. and Hamidekish, S.M. (2016) Molecular analysis of Sarcocystis spp. isolated from sheep (Ovis aries) in Babol area, Mazandaran Province, Northern Iran. Iran. J. Parasitol.,11(1): 73-80.

20. Daryani, A., Alaei, R., Dehghan, M., Arab, R., Sharif, M. and Ziaei, H. (2006) Survey of Sarcocystis infection in slaughtered sheep and buffaloes in Ardabil, Iran. J. Anim. Vet. Adv., 5(1): 60-62.

21. JyothiSree, C.H., Venu, R., Samatha, V., Malakondaiah, P. and Rayulu, V.C. (2017) Prevalence and microscopic studies of Sarcocystis infection in naturally infected water buffaloes (Bubalus bubalis) of Andhra Pradesh. J. Parasit. Dis., 41(2): 476-482.

22. Dubey, J. and Livingston, J.C. (1986) Sarcocystis capracanis and Toxoplasma gondii infections in range goats from Texas. Am. J. Vet. Res., 47(3): 523-524.

23. Prayson, B., McMahon, J.T. and Prayson, R.A. (2008) Fast food hamburgers: What are we really eating? Ann. Diagn. Pathol., 12(6): 406-409.

24. Avapal, R.S., Sharma, J. and Juyal, P. (2004) Pathological changes in Sarcocystis infection in domestic pigs (Sus scrofa). Vet. J., 168(3): 358-361.

25. Januškevičius, V., Januškevičienè, G., Banys, A., Dailidavičienė, J. and Aniulienè, A. (2018) Biochemical profile of blood in naturally occurring Sarcocystis infection 
in cattle. Acta Vet. Brno, 87(3): 205-211.

26. Dehaghi, M.M., Fallahi, M., Sami, M. and Radfar, M.H. (2013) Survey of Sarcocystis infection in slaughtered sheep in Kerman Abattoir, Kerman, Iran. Comp. Clin. Pathol., 22(3): 343-346.

27. Whaeeb, S.T. and Faraj, A.A. (2016) Molecular identification and phylogeny of microscopic Sarcocystis sheep in Baghdad province. Int. J. Adv. Res. Biol. Sci., 3(12): 50-56.

28. Abuelwafa, S.A., Alaraby, M.A., Abbas, I.E. and Elmishmishy, B.M. (2016) Prevalence of Sarcocystis species infecting sheep from Egypt. Egypt. Vet. Med. Soc. Parasitol. J., 12(7): 74-90.

29. Shekarforoush, S., Razavi, S., Dehghan, S. and Sarihi, K. (2005) Prevalence of Sarcocystis species in slaughtered goats in Shiraz, Iran. Vet. Rec., 156(13): 418-20.

30. Mavi, S.A., Teimouri, A., Mohebali, M., Yazdi, M.K.S., Shojaee, S., Rezaian, M., Salimi, M. and Keshavarz, H. (2020) Sarcocystis infection in beef and industrial raw beef burgers from butcheries and retail stores: A molecular microscopic study. Heliyon, 6(6): e04171.

31. Mounika, K., Chennuru, S., Ravipati, V., Tumati, S.R. and Krovvidi, S. (2018) Studies on prevalence and histomorphology of Sarcocystis species infecting cattle in Andhra Pradesh, India. J. Parasit. Dis., 42(1): 77-80

32. Shahraki, M.K., Ghanbarzehi, A. and Dabirzadeh, M. (2018) Prevalence and histopathology of Sarcocystosis in slaughtered carcasses in southeast Iran. J. Adv. Vet. Anim. Res., 5(4): 381-387.

33. Ahmed, A.M., Elshraway, N.T. and Youssef, A.I. (2016) Survey on Sarcocystis in bovine carcasses slaughtered at the municipal abattoir of El-Kharga, Egypt. Vet. World, 9(12): 1461-1465.

34. Nourani, H., Matin, S., Nouri, A. and Azizi, H. (2010) Prevalence of thin-walled Sarcocystis cruzi and thickwalled Sarcocystis hirsuta or Sarcocystis hominis from cattle in Iran. Trop. Anim. Health Prod., 42(6): 1225-1227.

35. Bahari, P., Salehi, M., Seyedabadi, M. and Mohammadi, A. (2014) Molecular identification of macroscopic and microscopic cysts of Sarcocystis in sheep in North Khorasan Province, Iran. Int. J. Mol. Cell Med., 3(1): 51-56.

36. Ferreira, M.S.T., Vogel, F.S.F., Sangioni, L.A., Cezar, A.S., Patrícia Braunig, P., Botton, S.A., Camillo, G. and Portella, L.P. (2018) Sarcocystis species identification in cattle hearts destined to human consumption in southern Brazil. Vet. Parasitol. Reg. Stud. Rep., 14: 94-98.

37. Imre, K., Dărăbuș, G., Tîrziu, E., Morariu, S., Imre, M., Plutzer, J., Boldea, M.V. and Morar, A. (2019) Sarcocystis spp. in Romanian slaughtered cattle: Molecular characterization and epidemiological significance of the findings. Biomed Res. Int., 2019: 4123154.

38. El-Dakhly, K.M., El-Nesr, K.A., El-Nahass, E.S., Hirata, A., Sakai, H. and Yanai, T. (2011) Prevalence and distribution patterns of Sarcocystis spp. in buffaloes in Beni-Suef, Egypt. Trop. Anim. Health Prod., 43(8): 1549-1554.

39. Davoudi, J., Shabestari, A.B., Mozahheb, P., Norouzi, R., Maadinia, M. and Mahmoudi, R. (2017) A study of Sarcocystis infection in mincemeat using digestion method in Ghazvin, Iran. Arch. Hyg. Sci., 6(2): 178-181.

40. Hu, J.J., Huang, S., Wen, T., Esch, G.W., Liang, Y. and Li, H.L. (2017) Sarcocystis spp. in domestic sheep in Kunming City, China: Prevalence, morphology, and molecular characteristics. Parasite, 24: 30.

41. Chiesa, F., Muratore, E., Dalmasso, A. and Civera, T. (2013) A new molecular approach to assess the occurrence of Sarcocystis spp. in cattle and products thereof: Preliminary data. Trop. Anim. Health Prod., 2(3): e41.

42. Rahdar, M. and Kardooni, T. (2017) Molecular identification of Sarcocystis spp. in sheep and cattle by PCR-RFLP from Southwest of Iran. Jundishapur J. Microbiol., 10(8): e12798. 